\title{
DECENTRALIZED NEURONAL CONTROL STRUCTURE
}

\author{
Victor H. Benitez, ${ }^{*}$ Edgar N. Sanchez ${ }^{*}$ \\ and Alexander G. Loukianov* \\ * CINVESTAV, Unidad Guadalajara, Apartado Postal 31-438, \\ Plaza la Luna, Guadalajara, Jalisco C.P. 45091, Mexico, \\ e-mail:vbenitez@gdl.cinvestav.mx.
}

\begin{abstract}
A novel decentralized variable structure neural control approach for large-scale uncertain systems is developed using Recurrent High Order Neural Networks (RHONN). It is assumed that each subsystem belongs to a class of block-controllable nonlinear systems whose vector fields includes interconnections terms. The interconnection terms are bounded by nonlinear functions. A decentralized RHONN structure and the respective learning law, are propposed in order to approximate on-line the dynamical behaviour of each nonlinear subsystem. The control law, which is able to regulate and to track the desired reference signals, is designed using the well known variable structure theory. The stability of the whole system is analyzed via the Lyapunov methodology. The applicability of proposed decentralized identification and control algorithm is illustrated via simulations as applied to stabilize an interconnected double inverted pendulum. Copyright $(2005$ IFAC
\end{abstract}

Keywords: Variable Structure Control, Nonliear Systems, Recurrent Networks, Large-scale Systems.

\section{INTRODUCTION}

The decentralized control approach often arises from the high dimension of the system to be controlled, the physical inability for subsystem information exchange, the lack of computing capabilities required for a single central controller and the uncertainty in measuring parameters values within a large-scale system [Lunze,1992]. A good example for such largescale systems is a multiarea power system with several power generating-units. It distributes electrical power over a wide geographical area and supplies many smaller and larger enterprises or privates homes with energy. Voltages and power flows at many different points have to be controlled. Obviously, no precise uniform model can be set up and no unique controller can be implemented for such a system. As a second example let consider a system of coupled water reservoirs whose levels have to be controlled so as to ensure sufficient reserves of water and, simultaneously, prevent the reservoirs from overflowing after a period of rain. Here, the difficulties of the control problems result from the complexity of the dynamical interactions between the reservoirs and the uncertainties concerning the amount of outflow to the consumer and inflow from the environment. Other examples of large-scale systems are traffic systems with complex dynamical behavior but relatively few measurement data and control inputs, large space structures with many different components, ecological systems with a large number of entities in close interactions, or coupled distillations columns and reactors in the chemical industry, steelrolling mills, flexible manufactory systems, or gas distribution networks [Lunze,1992]. All these examples illustrate either the lack of centralized information, or the lack of a centralized computing facility. These facts motivate the design of decentralized controllers, using only local information while guaranteeing sta- 
bility for the entire system. A detailed review for the development of decentralized adaptive control theory for large-scale dynamical systems is given in [Jiang, 1999]. The present paper introduces a new approach for decentralized control theory, introducing decentralized RHONN structures based on [Kosmatopoulos, 1997], which are able to identify the dynamical behaviour of subsystems with only local information and can deal with uncertainties in the absence of matching conditions, as have been demonstrated [Benitez, et al., 2003]. The Variable Structure Control Theory (VSC) is used to obtain a robust control law which guarantees tracking and rejects disturbances.

\section{A CLASS OF DECENTRALIZED SYSTEMS}

The considered large-scale system is constituted of nonlinear subsystems in the Nonlinear Block Controllable Form with Disturbance Term (NBC) [Loukianov, 1998]. The block $q$ for the $i^{\text {th }}(1 \leq i \leq N)$ subsystem is represented as follows

$$
\begin{aligned}
\dot{\chi}_{i}^{1}= & f_{i 1}\left(\chi_{i}^{1}\right)+B_{i 1}\left(\chi_{i}^{1}\right) \chi_{i}^{2}+\Gamma_{i 1 k}\left(\bar{\chi}_{k}^{1}\right) \\
\dot{\chi}_{i}^{2}= & f_{i 2}\left(\chi_{i}^{1}, \chi_{i}^{2}\right)+B_{i 2}\left(\chi_{i}^{1}, \chi_{i}^{2}\right) \chi_{i}^{3}+\Gamma_{i 2 k}\left(\bar{\chi}_{k}^{1}, \bar{\chi}_{k}^{2}\right) \\
& \vdots \\
\dot{\chi}_{i}^{q}= & f_{i q}\left(\chi_{i}^{1}, \chi_{i}^{2}, \ldots, \chi_{i}^{q}\right)+B_{i q}\left(\chi_{i}^{1}, \chi_{i}^{2}, \ldots, \chi_{i}^{q}\right) \chi_{i}^{r} \\
& +\Gamma_{i q k}\left(\bar{\chi}_{k}^{1}, \bar{\chi}_{k}^{2}, \ldots, \bar{\chi}_{k}^{q}\right), \quad q=3, \ldots, r-1 \\
\dot{\chi}_{i}^{r}= & f_{i r}(\chi)+B_{i r}(\chi) u_{i}+\Gamma_{i r k}(\bar{\chi})
\end{aligned}
$$

where $\chi=\left[\begin{array}{llll}\chi_{i}^{1 T} & \chi_{i}^{2 T} \ldots \chi_{i}^{r T}\end{array}\right]^{T}, \chi_{i}^{q} \in R^{n_{i q} \times 1} ; \bar{\chi}$ is the state vector of the $k^{t h}$ subsystem $(1 \leq k \leq N, k \neq i)$; the rank of $B_{i q}=n_{i q}, \forall \chi_{i}^{q} \in D_{\chi_{i}^{q}} \subset R^{n_{i q}}$. The interconnection terms

$$
\begin{aligned}
\Gamma_{i 1 k}= & \sum_{k=1, k \neq i}^{N} \gamma_{i 1 k}\left(\bar{\chi}_{k}^{1}\right) \\
\Gamma_{i 2 k}= & \sum_{k=1, k \neq i}^{N} \gamma_{i 2 k}\left(\bar{\chi}_{k}^{1}, \bar{\chi}_{k}^{2}\right) \\
& \vdots \\
\Gamma_{i q k}= & \sum_{k=1, k \neq i}^{N} \gamma_{i q k}\left(\bar{\chi}_{k}^{1}, \bar{\chi}_{k}^{2}, \ldots, \bar{\chi}_{k}^{q}\right) \\
\Gamma_{i r k}= & \sum_{k=1, k \neq i}^{N} \gamma_{i r k}(\bar{\chi})
\end{aligned}
$$

reflect the interaction between the $i^{t h}$ and $k^{t h}$ subsystem, are bounded by nonlinear functions $\gamma_{i r k}$ and enter the system as not matching condition disturbances, $f_{i r}$ and $B_{i r}$ are smooth and bounded functions, $f_{i r}(0)=0$ and $B_{i}(0)=0$. The integers $n_{i 1} \leq n_{i 2} \leq \ldots \leq n_{i r}$ defines the different subsystem structures, and $\sum_{q=1}^{r} n_{i q}=n_{i}$.

\section{DECENTRALIZED NEURAL NETWORK}

In this section we extend the recurrent neural network developed in [Benitez, 2003, and references within] to the decentralized control problem; additionally, we present the decentralized recurrent neural network which is able to identify (1).

For the large-scale plant, described by (1), there exists a decentralized RHONN block controllable structure as follows

$$
\begin{aligned}
& \dot{x}_{i}^{1}=-A_{i 1} x_{i}^{1}+\sum_{p=1}^{L_{1 p}} w_{i 1 p} \prod_{j \in I 1 p} Z_{1 p}^{d_{1 j}(p)} \\
& +\sum_{m=L_{1 p}+1}^{L_{1 p}^{\prime}} w_{i 1 m}^{\prime} \Psi_{i 1}\left(x_{i}^{1}\right) x_{i}^{2} \\
& \dot{x}_{i}^{2}=-A_{i 2} x_{i}^{2}+\sum_{p=1}^{L_{2 p}} w_{i 2 p} \prod_{j \in I 2 p} Z_{2 p}^{d_{2 j}(p)} \\
& +\sum_{m=L_{2 p}+1}^{L_{2 p}^{\prime}} w_{i 2 m}^{\prime} \Psi_{i 2}\left(x_{i}^{1}, x_{i}^{2}\right) x_{i}^{3} \\
& \vdots \\
& \dot{x}_{i}^{q}=-A_{i q} x_{i}^{q}+\sum_{p=1}^{L_{q p}} w_{i q p} \prod_{j \in I q p} Z_{q p}^{d_{q j}(p)} \\
& +\sum_{m=L_{q p}+1}^{L_{q p}^{\prime}} w_{i q m}^{\prime} \Psi_{i q}\left(x_{i}^{1}, x_{i}^{2}, \ldots, x_{i}^{q}\right) x_{i}^{r} \\
& q=3, \ldots, r-1 \\
& \dot{x}_{i}^{r}=-A_{i r} x+\sum_{p=1}^{L_{r p}} w_{i r p} \prod_{j \in I r p} Z_{r p}^{d_{r j}(p)} \\
& +\sum_{m=L_{r p}+1}^{L_{r p}^{\prime}} w_{i r m}^{\prime} \Psi_{i r}(x)
\end{aligned}
$$

where $x=\left[\begin{array}{llll}x_{i}^{1 T} & x_{i}^{2 T} \cdots & x_{i}^{r T}\end{array}\right]^{T}$ is the $i^{t h}$ block neuron state with the same properties than (1); $L$ is the number of high order connections, $L^{\prime}$ is the number of fixed parameters $w^{\prime}$, which depends on the plant structure and are incorporated to the neural network model in order to obtain a block controllable structure, $\left\{I_{1 p}, I_{2 p}, \ldots, I_{r p}\right\}$ is a collection of no ordered subsets of $\{1 p, 2 p, \ldots, m+n\}, A$ is a diagonal matrix with positive entries, $w$ are the adjustable weights of the neural network, $d$ are no negative integers, $\Psi$ is a nonlinear function of the state $x$ and/or the input $u$, and $Z$ is a vector defined as

$$
Z_{p}=\left[\begin{array}{c}
S_{i}\left(x_{i}^{1}\right) \\
\vdots \\
S_{i}\left(x_{i}^{r}\right)
\end{array}\right]
$$

with $S_{i}(\cdot)$ as a smooth sigmoid function formulated by

$$
S_{i}(x)=\frac{\alpha_{i}}{1+\exp \left(-\beta_{i} x\right)}-\gamma_{i}
$$


where $S_{i}(\cdot) \in[-1,1] ; \alpha, \beta$ and $\gamma$ are positive constants. If the following vector is introduced for the $i^{t h}$ subsystem

$$
\rho_{i}\left(x_{i}, u_{i}\right)=\left[\begin{array}{c}
\rho_{i, 1} \\
\rho_{i, 2} \\
\vdots \\
\rho_{i, L_{p}}
\end{array}\right]=\left[\begin{array}{c}
\prod_{j \in I i 1} Z_{1}^{d_{1 j}(i 1)} \\
\prod_{j \in I i 2} Z_{2}^{d_{2 j}(i 2)} \\
\vdots \\
\prod_{j \in I i r} Z_{r}^{d_{r j}(i r)}
\end{array}\right]
$$

then, system (3) can be simplified as

$$
\begin{aligned}
\dot{x}_{i j} & =-a_{i j} x_{i j}+w_{i}^{T} \rho_{i}\left(x_{i}, u_{i}\right)+w_{i}^{\prime T} \psi_{i}(x, u) x_{j+1} \\
j & =1, \ldots, r-1
\end{aligned}
$$

In (5), the $j^{t h}$ state of each neuron for the $i^{t h}$ subsystem is characterized by $x$, and $\psi$ denotes a nonlinear function of $x$ or $u$ which are designed according to the plant structure.

Remark 1. It is worth to notice that (3) does not consider the interconection terms.

\subsection{On-Line Learning Law}

The decentralized identification and control scheme proposed is based only on local information available for each subsystem. Hence, the learning law is developed for the subsystem $i$ as follows.

Based on the results presented in [Kosmatopoulos, 1997], [Sanchez, 2000], we assume that there exists a decentralized RHONN which fully describes (1), thereby the plant model can be described by

$$
\dot{\chi}_{i}=-A_{i} \chi_{i}+w_{i}^{* T} \rho_{i}\left(\chi_{i}, u_{i}\right)+\nu_{i}(t)
$$

where $w^{*} \in R^{L_{i r}}$ and the modelling error $\nu_{i}(t)$ is given by

$$
\begin{aligned}
\nu_{i}(t)= & f_{i}\left(\chi_{i}\right)+B_{i}\left(\chi_{i}\right) u_{i}+\Gamma_{i 1 k}\left(\bar{\chi}_{k}\right) \\
& +A_{i} \chi_{i}-w_{i}^{T} \rho_{i}\left(\chi_{i}, u_{i}\right)
\end{aligned}
$$

The modeling error term $\nu_{i}(t)$ can be made arbitrary small selecting appropriately the number $L$ of high order connections [Kosmatopoulos et al., 1995]. The optimal unknown weights vector $w^{*}$ is defined as

$$
w_{i}^{*}=\arg \min _{w_{i}}\left\{\sup _{\chi_{i, u_{i}}}\left|f_{i}+B_{i} u_{i}+A_{i} \chi_{i}-w_{i}^{T} \rho_{i}\right|\right\}
$$

Since each subsystem (1) can be written according to (6), two possible models for (5) can be used:

- Parallel model

$$
\dot{x}_{i j}=-a_{i j} x_{i j}+w_{i}^{T} \rho_{i}\left(x_{i}, u_{i}\right)+w_{i}^{\prime T} \psi_{i}(x, u)
$$

- Series-Parallel model

$$
\dot{x}_{i j}=-a_{i j} x_{i j}+w_{i}^{T} \rho_{i}\left(\chi_{i}, u_{i}\right)+w_{i}^{\prime T} \psi_{i}(\chi, u)
$$

In this paper we use the Series-Parallel one.

\subsection{On-Line Weight Update Law}

In this subsection, a learning law is developed considering the case where the modeling error term is zero, i.e., $\nu_{i}(t)=0$. From (6), for the $j^{t h}$ state, we establish

$$
\begin{aligned}
\dot{\chi}_{i j} & =-a_{i j} \chi_{i j}+w_{i j}^{* T} \rho_{i j}, \\
i & =1, . ., N \\
j & =1, \ldots, n_{i q}
\end{aligned}
$$

and considering (8) or (9) then

$$
\begin{aligned}
\dot{x}_{i j} & =-a_{i j} x_{i j}+w_{i}^{T} \rho_{i j} \\
i & =1, . ., N, \\
j & =1, \ldots, n_{i q}
\end{aligned}
$$

where $w_{i}$ is the estimated of the unknown vector $w_{i}^{*}$. In this case the state error $e_{i j} \triangleq x_{i j}-\chi_{i j}$ satisfies

$$
\begin{aligned}
\dot{e}_{i j} & =-a_{i j} e_{i j}+\phi_{i j}^{T} \rho_{i j} \\
i & =1, . ., N \\
j & =1, \ldots, n_{i q}
\end{aligned}
$$

with $\phi_{i j}=w_{i j}^{T}-w_{i j}^{*}$. The following theorem establishes the learning law which is able to on-line adjust the weights for neural network (11) as well as the stability and convergence properties.

Theorem 1. Consider the RHONN model given by (11) whose weights are adjusted according to

$$
\dot{w}_{i j}=-e_{i j} \Xi_{i j}^{-1} \rho_{i j}
$$

where $\Xi_{i j}^{-1}$ is a symmetric positive definite matrix, then for $i=1, \ldots, N$ and $j=1, \ldots, n_{i q}$

(a) $\mathrm{e}_{i j}, \phi_{i j} \in \mathcal{L}_{\infty}$

(b) $\lim _{t \rightarrow \infty} e_{i j}(t)=0$

The proof is given in [Rovithakis, 2000].

\subsection{Robust Updating Weight Law}

For the case where the modelling error is not zero [Rovithakis, 2000], the solutions of differential equations (13) may become unbounded, even if the modelling error is bounded. Therefore, the learning law (13) has to be modified in order to avoid the parameter drift problem. The learning laws given by (13) are modified as follows

$$
\dot{w}_{i j}=-\Xi_{i j}^{-1}\left(e_{i j} \rho_{i j}-\sigma_{i j} w_{i j}\right)
$$

where

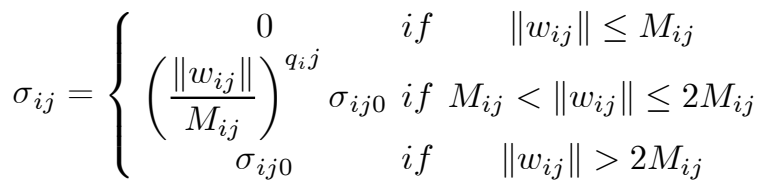

with $q \geq 1, \sigma_{i j 0}$ and $M_{i j}$ positive constants. 


\section{CONTROL LAW}

Once, the large-scale system is modelled by the proposed neural identifier, we proceed to develop the respective control law.

\subsection{Block Control Algorithm}

A sliding surface and a discontinuous control law is designed for the system (5) considering the state $x_{i}^{q+1}$, $q=3, \ldots, r-1$ as a fictitious control vector in the block $q$. This procedure is described in the next steps [Loukianov, 1998]

Step 1 Assume that $n_{i 1}=n_{i 2}$ and define the vector error as

$$
z_{i}^{1}=x_{i}^{1}-\delta_{i}
$$

where $\delta_{i}$ is a smooth and bounded reference signal. The dynamics for (15) in the trajectories of (5) are

$$
\begin{aligned}
\dot{z}_{i}^{1}= & -a_{i} x_{i}+w_{i}^{T} \rho_{i}\left(x_{i}, u_{i}\right) \\
& +w_{i}^{\prime T} \psi_{i}(x, u) x_{2}-\dot{\delta}_{i}
\end{aligned}
$$

If the fictitious control $x_{i}^{2}$ is selected as

$$
\begin{aligned}
x_{i}^{2} & =\mu_{i}\left(-k_{i 1} z_{i}^{1}+z_{i}^{2}+\dot{\delta}_{i}+a_{i} x_{i}-\eta_{i}\right) \\
\mu_{i} & =\left(w_{i}^{\prime T} \psi_{i}(x, u)\right)^{-1} \\
\eta_{i} & =w_{i}^{T} \rho_{i}\left(x_{i}, u_{i}\right)
\end{aligned}
$$

where $k$ is a constant positive, then, the first block is transformed in the new coordinates $z_{i}^{1}, z_{i}^{2}$ as follows

$$
\dot{z}_{i}^{1}=-k_{i 1} z_{i}^{1}+z_{i}^{2}
$$

The vector $z_{i}^{2}$ is obtained using (17) as

$$
\begin{aligned}
z_{i}^{2}= & -a_{i} x_{i}+w_{i}^{T} \rho_{i}\left(x_{i}, u_{i}\right) \\
& +w_{i}^{\prime T} \psi_{i}(x, u) x_{2}-\dot{\delta}_{i}+k_{i 1} z_{i}^{1} \\
z_{i}^{2} \triangleq & \alpha_{i}^{1}\left(x_{i 1}, x_{i 2}\right)
\end{aligned}
$$

Step 2 Taking the derivative of (19) we obtain

$$
\dot{z}_{i}^{2}=\frac{\partial \alpha_{i}^{1}}{\partial x_{i 1}} \dot{x}_{i 1}+\frac{\partial \alpha_{i}^{1}}{\partial x_{i 2}} \dot{x}_{i 2}-\ddot{\delta}_{i}
$$

The fictitious control for (21) is $x_{i}^{3}$ and the procedure is successive for the remainder of the blocks until the true control $u_{i}$ is obtained in the $r^{\text {th }}$ block

$$
\dot{z}_{i}^{r}=f_{i r}-\xi_{i r} u_{i}
$$

where the rank of $\xi_{i r}=n_{i r}$ and $f_{i r}$ is a bounded function. The discontinuous control action is applied as

$$
u_{i}=U_{i 0} \operatorname{sign}\left(z_{i}^{r}\right)
$$

\subsection{Stability Analysis}

Let define

$$
B_{i}=\left\{f_{i r} \in R^{n_{i r}} \mid\left\|f_{i r}\right\|<\epsilon_{i}\right\}
$$

as a ball of radius $\epsilon$. Setting $U_{i 0}$ in (23) as

$$
U_{0 i}>\frac{\left(\epsilon_{i}+\alpha_{i 0}\right)}{\xi_{i r}}
$$

with $\alpha_{i 0}$ a positive constant. Let consider the candidate Lyapunov function $v_{i}=\frac{1}{2} z_{i r}^{T} z_{i r}$, whose derivative in the trajectories of (22) are obtained as

$$
\dot{v}_{i}=z_{i r}^{T}\left(f_{i r}-\xi_{i r} U_{i 0} \operatorname{sign}\left(z_{i r}\right)\right)
$$

Taking into account the following identities

$$
z^{T} \operatorname{sign}(z)=\|z\|_{1} \geq\|z\|_{2}
$$

and substituting them in (26), we obtain

$$
\begin{aligned}
& \dot{v}_{i} \leq\left\|z_{i r}\right\|\left\|f_{i r}\right\|-\xi_{i r} U_{0 i}\left\|z_{i r}\right\| \\
& \dot{v}_{i} \leq-\left\|z_{i r}\right\|\left(-\left\|f_{i r}\right\|+\xi_{i r} U_{i 0}\right)
\end{aligned}
$$

If (24) holds and using (25) in (27) the derivative of the Lyapunov function is simplified as

$$
\dot{v}_{i} \leq-\left\|z_{i r}\right\| \alpha_{i 0}
$$

Consequently, the closed loop asymptotic stability is guarantee for (22) and the sliding motion occurs on the manifold $z_{i}^{r}=0$ in a finite time, then the tracking error (15) will tends asymptotically to zero in accordance with (18). Moreover, the composite Lyapunov function candidate for the large-scale system $V=\sum_{i=1}^{N} v_{i}$ whose derivative

$$
\dot{V} \leq-\left\|z_{i r}\right\| \sum_{i=1}^{N} \alpha_{i 0}
$$

is negative defined, guarantees the stability for the global interconnected system.

\section{TWO CONNECTED PENDULUM EXAMPLE}

In this section, we present an illustrative benchmark example for the identification and control scheme applied to a two inverted pendulums connected by a spring, which is a classical large-scale testbed for nonlinear decentralized control [Spooner, 1999], [Gavel, 1989], [Nardi, 2001].

\subsection{Description}

Each pendulum is positioned by a torque input $u_{i}$ applied to a servomotor at its base. It is assumed that, for the $i^{t h}$ controller $(i=1,2)$, the only available measuraments are $\chi_{i 1}$ and $\dot{\chi}_{i 1}$ (angular position and rate).

The equations which describe the motion of each pendulum are defined by 


$$
\begin{aligned}
\dot{\chi}_{i 1}= & \chi_{i 2} \\
\dot{\chi}_{i 2}= & \left(\frac{m_{i} g r}{J_{i}}-\frac{k r^{2}}{4 J_{i}}\right) \sin \left(\chi_{i 1}\right)+\frac{k r}{2 J_{i}}(l-b) \\
& +\frac{u_{i}}{J_{i}}+\frac{k r^{2}}{4 J_{i}} \gamma_{i 2} \\
\gamma_{i 2}= & \begin{cases}\sin \left(\chi_{21}\right) & \text { if } i=1 \\
\sin \left(\chi_{11}\right) & \text { if } i=2\end{cases} \\
i= & 1,2
\end{aligned}
$$

where $\chi_{i 1}$ is the angular displacement of the pendulums from the vertical. The parameters are as follows

$$
\begin{array}{cc}
m_{1} & 2 \\
m_{2} & 2.5 \\
J_{1} & 0.5 \\
J_{2} & 0.625 \\
k & 100 \\
r & 0.5 \\
l & 0.5 \\
g & 9.81 \\
b & 0.4
\end{array}
$$

Table 1: Double inverted pendulum parameters

For the plant dynamics (28)-(30), we propose the decentralized neural network identifiers, according to the series-parallel model (9) as

$$
\begin{aligned}
\dot{x}_{i 1}= & x_{i 2} \\
\dot{x}_{i 2}= & -a_{i 2} x_{i 2}+w_{i 21} s_{i}\left(\chi_{i 1}\right) \\
& +w_{i 22} s_{i}\left(\chi_{i 2}\right)+\frac{u_{i}}{J_{i}} \\
i= & 1,2
\end{aligned}
$$

The goal is to track desired reference signals, which is achieved by designing a control law based on the sliding mode technique. Consider the reference signal as $\delta_{i}$, for $i=1,2$, then the control error is given by

$$
z_{i 1}=x_{i 1}-\delta_{i}
$$

whose dynamics are obtained using (31) as

$$
\dot{z}_{i 1}=x_{i 2}-\dot{\delta}_{i}
$$

Introducing new dynamics for (33) as

$$
\dot{z}_{i 1}=-k_{i 1} z_{i 1}+z_{i 2}
$$

the dynamics for new variables $z_{i 2}$ is obtained as

$$
\dot{z}_{i 2}=f_{i 2}+\frac{1}{J_{i}} u_{i}
$$

where

$$
\begin{aligned}
f_{i 2}= & -a_{i 2} x_{i 2}+w_{i 21} s_{i}\left(\chi_{i 1}\right)+w_{i 22} s_{i}\left(\chi_{i 2}\right)(36) \\
& -\ddot{\delta}_{i}-k_{i 1}^{2} z_{i 1}+k_{i 1} z_{i 2}
\end{aligned}
$$

Then, the control action is proposed, for each pendu$\operatorname{lum}(i=1,2)$, as

$$
u_{i}=-U_{i 0} \operatorname{sign}\left(z_{i 2}\right)
$$

\subsection{Simulation results}

This subsection presents the respective simulation results. Fig.1 display the regulation case with a $10 \mathrm{~N}-\mathrm{m}$ torque disturbance applied at 3 sec. Fig.2 and Fig.3 shows the control law.

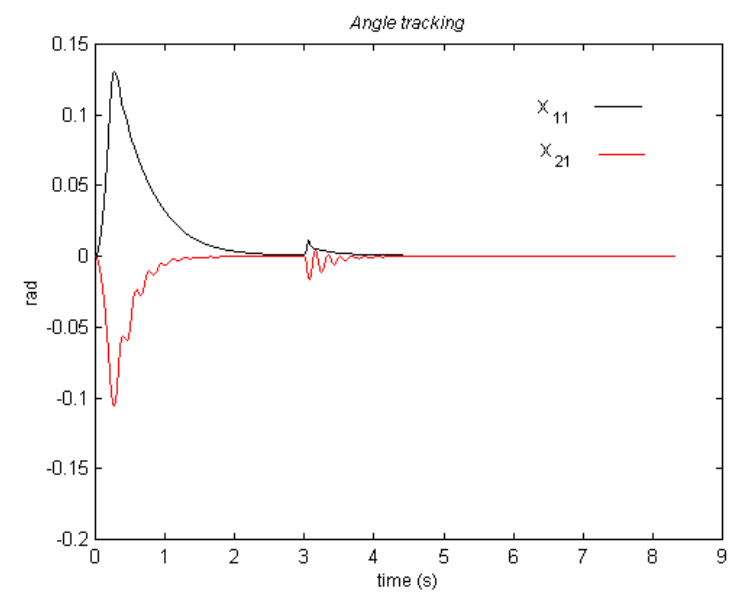

Fig. 1. Regulation

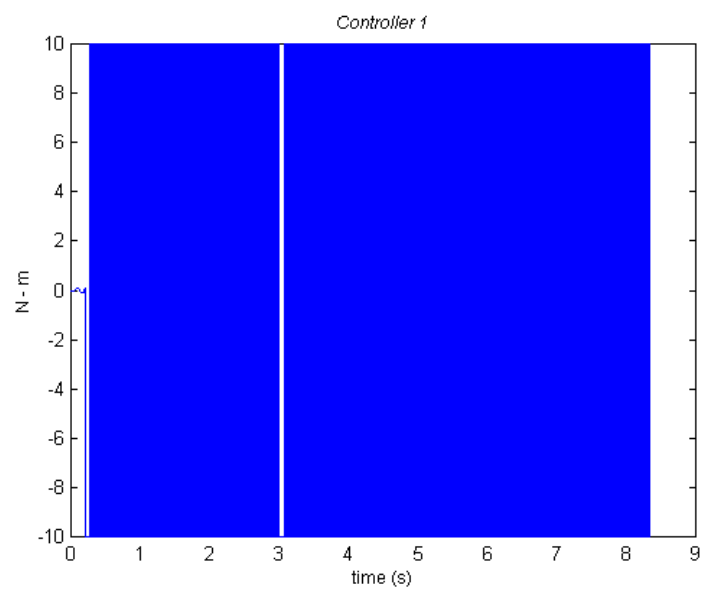

Fig. 2. Control action for controller 1

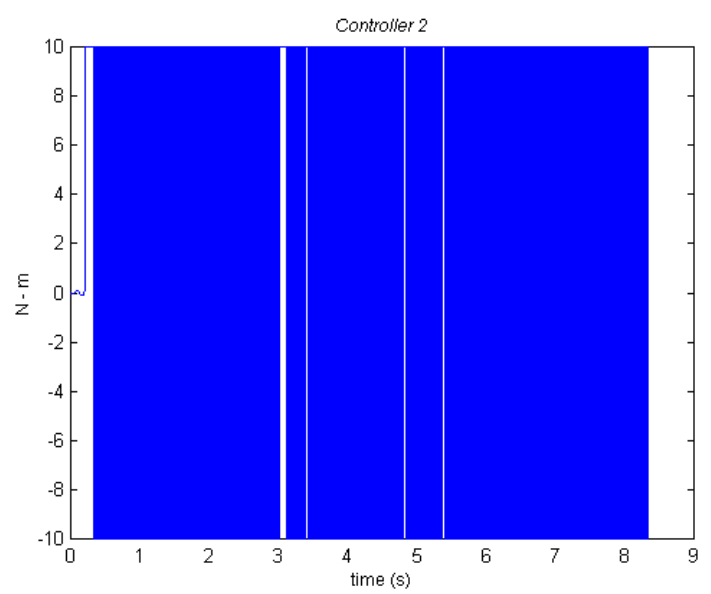

Fig. 3. Control action for controller 2

Figs. 4-6 displays different reference signals and the respective tracking. In Fig. 4, a sine reference for both pendulums is tracked, Fig. 5 shows the tracking for a sine and cosine references for positions $\chi_{11}$ and $\chi_{21}$ respectively. Finally, a more complex behaviour 
is displayed in Fig. 6, where $\chi_{11}$ is kept fixed whereas $\chi_{21}$ tracks a sine signal.

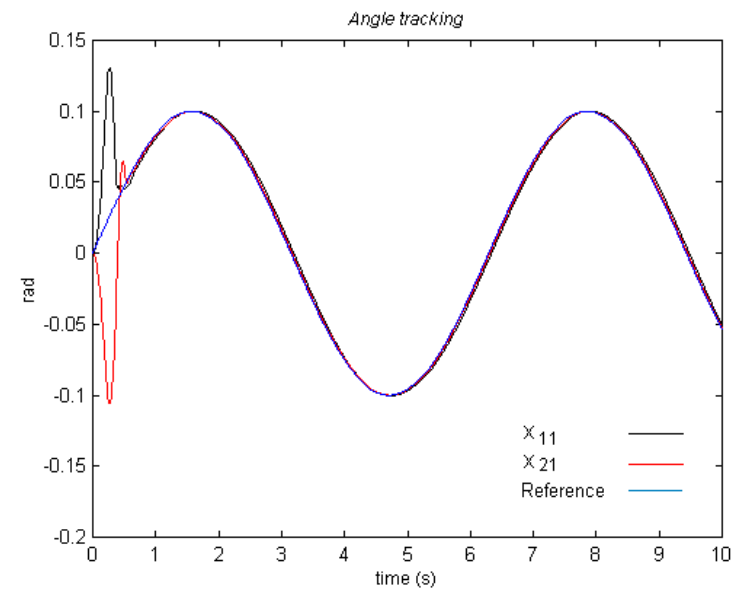

Fig. 4. Tracking for a sine reference

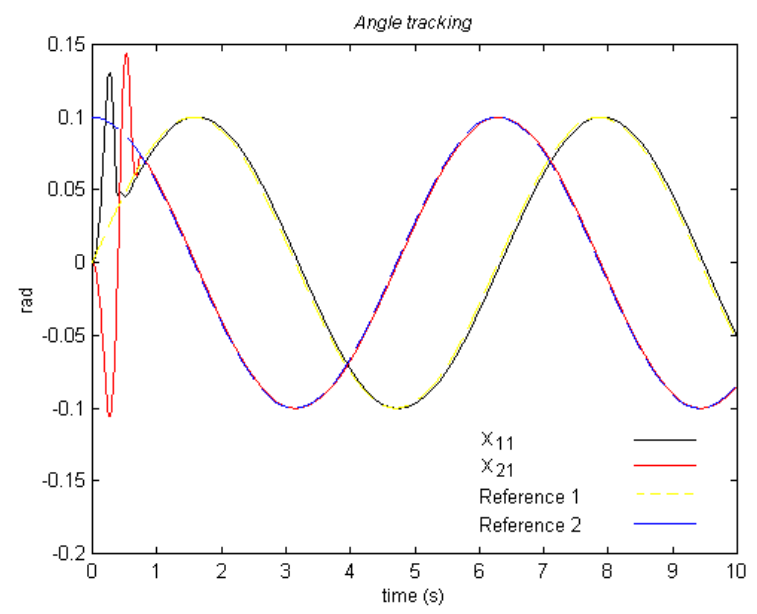

Fig. 5. Tracking for a sine and cosine reference signal

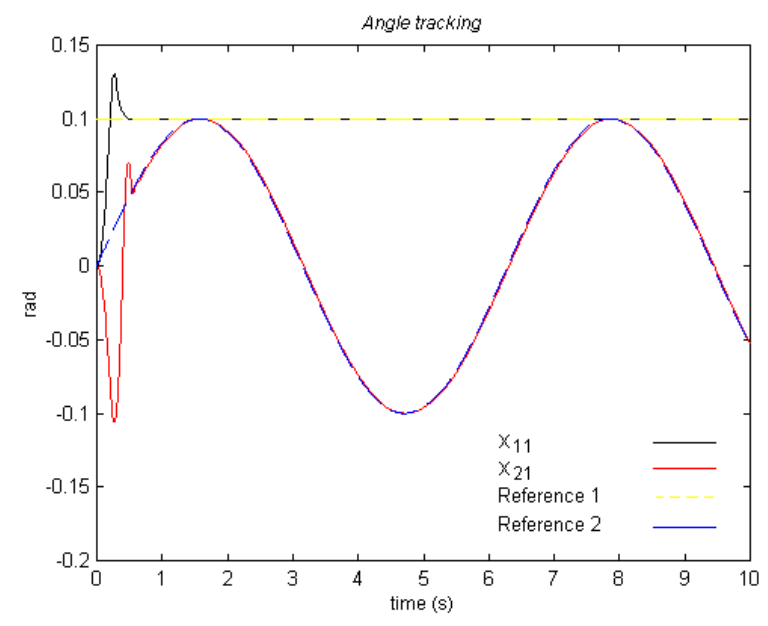

Fig. 6. Tracking for constant and sine refereces

\section{CONCLUSIONS}

A decentralized identification and control scheme is proposed which is able to identify each subsystem dynamics for a double inverted pendulum. The sliding mode control law forces the closed loop trajectory to converge and to stay in the sliding manifold, which guarantees that the tracking error is zero. Moreover, simulations illustrates that the scheme proposed preserves stability and presents good performance.

\section{REFERENCES}

Benitez, V.H., A.G. Loukianov and E.N. Sanchez (2003). Neural identification and control of a linear induction motor using an alpha-beta model. In: Proceedings of the American Control Conference. Denver, Colorado, USA. pp. 4041-4046.

Gavel, D.T. and D.D. Siljak (1989). Decentralized adaptive control: structural conditions for stability. IEEE Transactions on Automatic Control 34(34), 413-426.

Jiang, Z.P. (1999). New results in decentralized adaptive nonlinear control with output feedback. In: Proceedings of the 38th Conference on Decision and control. Phoenix, Arizona, USA. pp. 47724777.

Kosmatopoulus, E.B., M.A. Christodoulou and P.A. Ioannou (1997). Dynamical neural network that ensure exponential identification error convergence. Neural Networks 10(2), 299-314.

Kosmatopoulus, E.B., M.M. Polycarpou, and P.A. Ioannou (1995). Higher order neural network structures for identification of dynamical systems. IEEE Transactions on Neural Networks 6(2), 422-431.

Loukianov, A.G. (1998). Nonlinear block control with sliding mode. Automation and Remote Control 57(7), 916-933.

Lunze, J. (1992). Feedback Control of Large-Scale Systems. Prentice Hall. UK.

Nardi, F., N. Hovakimyan and A.J. Calise (2001). Decentralized control of large-scale systems using single hidden layer neural networks. In: Proceedings of the American Control Conference. Vol. 4. Arlington, Virginia, USA. pp. 916-933.

Rovithakis, G.A. and M.A. Christodoulou (2000). Adaptive Control with Recurrent High-Order Neural Networks. Springer-Verlag. London, England.

Sanchez, E.N., A.G. Loukianov and R.A. Felix (2000). Stepper motor trajectory tracking combining dynamic triangular neural networks and sliding modes. In: 6th International Workshop on Variable Structure Systems. Gold Coast Australia. pp. 916-933.

Spooner, J.T. and K.M. Passino (1999). Decentralized adaptive control of nonlinear systems using radial basis neural networks. IEEE Transactions on Automatic Control 44(11), 2050-5057. 